„DIE DEUTSCHE COVID-19 OMICS INITIATIVE WURDE IM MÄRZ 2020

GESTARTET - MIT SEQUENCING TECHNOLOGIEN UND FUNCTIO-

NAL GENOMICS WIRD DAS WISSEN ÜBER DAS SARS-COV-2-VIRUS,

DIE URSACHEN SCHWERER KRANKHEITSVERLÄUFE UND DIE IMMUN-

ANTWORT AUF COVID-19 VORANGETRIEBEN.“

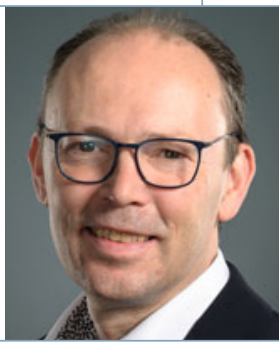

Joachim L. Schultze

\title{
Deutsche COVID-19 Omics Initiative (DeCOI)
}

DOI: $10.1007 / \mathrm{s} 12268-021-1586-4$

(C) Der Autor 2021

- Nach über einem Jahr Pandemie wissen wir, wie wichtig wissenschaftlicher Erkenntnisgewinn für die Krisenbewältigung ist. Für die Bevölkerung ist dies am Beispiel der schnellen Erforschung der Impfstoffe am leichtesten $\mathrm{zu}$ begreifen. Aber andere Wissenschaftsbereiche haben einen nicht minder wichtigen Anteil an der Krisenbewältigung. Hier kommt der Genomforschung eine Schlüsselrolle $\mathrm{zu}$. Ohne Sequenzierung keine Entschlüsselung der Erbinformation des Virus. Ohne Erbinformation keine Impfung und keine molekulargenetische Surveillance zur Identifikation und Verbreitung gefährlicher Mutanten. Ohne Sequenzierung kein Hinweis auf Mutationen in für die Virusabwehr essenziellen menschlichen Genen.

In einer Bottom-Up-Bewegung trieben ins besondere die Mitglieder des German Human Genome Archive (GHGA) Konsortiums und des Netzwerkes der DFG-geförderten NGS-Kompetenzzentren (NGS-CN) am Anfang die Aktivitäten voran. Mitte März 2020 baten die NGS-CNs die DFG um Unterstützung nachdem die Universitäten der NGS-CNs in Kiel, Dresden, Tübingen, Saarbrücken, Bonn, Köln und Düsseldorf bereits der Öffnung der Sequenzier-Einrichtungen im Lockdown zugestimmt hatten. Am 30. März gab auch die DFG grünes Licht. Bereits wenige Tage zuvor hatten sich Mitglieder aus den NGS-CN, GHGA und vieler anderer Institute in Deutschland zur Deutschen COVID-19 OMICS Initiative (DeCOI, https://decoi.eu) zusammengeschlossen.

Im Sinne des schnellstmöglichen Erkenntnisgewinns war es essenziell, jegliche für den normalen Wissenschaftsbetrieb übliche Kompetition zwischen den Arbeitsgruppen durch das Prinzip des Teilens von Wissen, Expertise, SOPs, Analyse-Pipelines, Ethik-Dokumenten, Proben, Daten und Analyse-Kapazitäten zu ersetzen. Diese Team-orientierte Vorgehensweise hat DeCOI die entsprechende Geschwindigkeit ermöglicht und hat damit auch im internationalen Vergleich zeigen können, zu welchen Höchstleistungen Wissenschaftler an deutschen Institutionen gemeinsam in der Lage sind. Heute gehören DeCOI über 100 Wissenschaftler an mehr als 50 Institutionen in ganz Deutschland an. Interessierte Wissenschaftler sind herzlich eingeladen, bei DeCOI mitzuwirken.

Bereits im Mai 2020 waren die DeCOI-Mitglieder, die sich mit Virus-Sequenzierung beschäftigen, in der Lage, Protokolle durchzuführen, mit denen eine molekulargenetische
Surveillance für Deutschland möglich gemacht werden konnte. Heute sind diese Kollegen Teil der molekulargenetischen Surveillance in Deutschland, arbeiten eng mit dem RKI zusammen [1,2], und sind als Teil des B-FASTProjektes im Netzwerk Universitätsmedizin (NUM) auch Bestandteil der vom BMBF geförderten föderierten Struktur zur PandemieAbwehr. Gemeinsam mit vielen engagierten Kollegen an Universitäten, Helmholtz-Zentren, Max-Planck-, Leibniz- und Fraunhofer-Instituten bauen sie zurzeit die akademische Infrastruktur für eine flächendeckende und hoffentlich auch nachhaltige Molekulargenetische Surveillance für Deutschland auf, alles mit dem Ziel, für zukünftige Epidemien und Pandemien besser gewappnet zu sein.

In mehreren, hochrangig publizierten Arbeiten [3-5] haben DeCOI-Mitglieder zeigen können, dass die moderne funktionelle Genomik inklusive der Einzelzellgenomik durch ihre Daten- und Hypothesen-getriebene Vorgehensweise klassischen Methoden der Immunologie bei der schnellstmöglichen und umfassenden Beschreibung einer neuen Erkrankung wie COVID-19 weit überlegen ist. Hier hat DeCOI auch weltweit eine Vorreiter-Rolle eingenommen, indem das Prinzip der Mehr-KohortenEinzelzell-Genom-Projekte erstmalig im klinischen Kontext zur Anwendung kam.

Die Errungenschaften von DeCOI erstrecken sich auf weitere zukunftsträchtige Forschungsbereiche. So leisten DeCOI-Mitglieder in internationalen Konsortien zur genetischen Suszeptibilität bei schweren COVID-19-Erkrankungen einen wichtigen Beitrag. Aber auch die erstmalige Anwendung völlig neuer KI-Konzepte, wie das Swarm Learning zur Identifikation von COVID-19-Patienten mittels Blut-TranskriptomAnalysen, war nur durch den unermüdlichen Einsatz vieler DeCOI-Mitglieder überhaupt möglich [6].

Mit DeCOI hat die Genomforschung gezeigt, welche außerordentliche Stellung sie in der Krisenabwehr einnehmen kann. Es ist an der Zeit, dass die Ministerien ernsthaft über föderierte, institutionalisierte Strukturen für die deutsche Genomforschung nachdenken und zu deren Umsetzung ihren Beitrag leisten. Die Genomforscher in Deutschland sind bereit dazu.

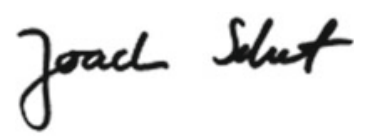

Joachim L. Schultze

Direktor Systemmedizin und PRECISE Platform for Single Cell Genomics and Epigenomics

\section{Danksagung}

Ich bedanke mich bei allen Mitgliedern von DeCOI für die exzellente Zusammenarbeit, die vielen gemeinsamen Stunden, die wir gemeinsam mit Wissenschaft verbracht haben, die Offenheit und der unerschütterliche Wille, gemeinsam einen kleinen Beitrag zur Bewältigung der Krise zu leisten.

Lesen Sie mehr zu DeCOI in der nächsten Ausgabe von BIOspektrum.

\section{Literatur}

[1] van der Toorn W, Oh D-Y, von Kleist M, working group on SARS-CoV-2 Diagnostics at RKI (2021)

COVIDStrategyCalculator: A software to assess testing- and quarantine strategies for incoming travelers, contact person management and de-isolation. Patterns (N Y) 100264 [2] van der Toorn W, Oh D-Y, Bourquain D et al. (2021) An intra-host SARS-CoV-2 dynamics model to assess testing and quarantine strategies for incoming travelers, contact person management and de-isolation. Patterns (N Y) 100262 [3] Schulte-Schrepping J, Reusch N, Paclik D et al. (2020) Severe COVID-19 Is Marked by a Dysregulated Myeloid Cell Compartment. Cell 182:1419-1440.e23

[4] Aschenbrenner AC, Mouktaroudi M, Krämer B, et al. (2021) Disease severity-specific neutrophil signatures in blood transcriptomes stratify COVID-19 patients. Genome Med 13:7

[5] Bernardes JP, Mishra N, Tran F et al. (2020) Longitudinal Multi-omics Analyses Identify Responses of Megakaryocytes, Erythroid Cells, and Plasmablasts as Hallmarks of Severe COVID-19. Immunity 53:1296-1314.e9

[6] Warnat-Herresthal S, Schultze H, Shastry KL et al. (2020) Swarm Learning as a privacy-preserving machine learning approach for disease classification. BioRxiv

Funding note: Open Access funding enabled and organized by Projekt DEAL. Open Access: Dieser Artikel wird unter der Creative Commons Namensnenn 4.0 International Lizenz veröffentlicht, welche die Nutzung, Vervielfältigung, Bearbeitung, Verbreitung und Wiedergabe in jeglichem Medium und For erlaubt, sofern Sie den/die ursprünglichen Autor(en) und die Quelle

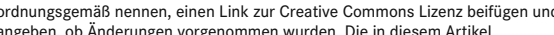
enthaltenen Bilder und sonstiges Drittmaterial unterliegen ebenfalls der genannten Creative Commons Lizenz, sofern sich aus der Abbildungslegende nichts anderes ergibt. Sofern das betreffende Material nicht unter der genannten Creative Commons Lizenz steht und die betreffende Handlung nicht nach gesetzlichen Vorschriften erlaubt ist, ist für die oben aufgeführten Weiterverwendungen des Materials die Einwilligung des jeweiligen Rechteinhabers einzuholen. Weitere Details zur Lizenz entnehmen Sie bitte der
Lizenzinformation auf http://creativecommons.org/licenses/by/4.0/deed.de.

\section{Korrespondenzadresse}

Prof. Dr. med. Joachim L. Schultze

Direktor Systemmedizin und PRECISE Platform for Single Cell Genomics and Epigenomics Deutsches Zentrum für Neurodegenerative

Erkrankungen e.V. (DZNE)

Venusberg-Campus 1/99

D-53127 Bonn

Genomik \& Immunregulation

Life \& Medical Sciences (LIMES) Institut

Universität Bonn

joachim.schultze@dzne 